\title{
Evaluación del perfil competencial de alumnos universitarios respecto a la educación tecnológica
}

\section{Evaluation of the competence profile of University students regarding educational technology}

\author{
María Dolores Díaz Durán ${ }^{1}$, Juan Jesús Martín Jaime ${ }^{2}$ \\ ${ }^{1}$ Facultad de Ciencias de la educación, Universidad de Málaga, España (diazduran@uma.es) \\ ${ }^{2}$ Facultad de Ciencias de la educación, Universidad de Málaga, España (jjmartin@uma.es)
}

Recibido el 13 de abril de 2015; revisado el 19 de abril de 2015; aceptado el 21 de abril de 2015; publicado el 5 de junio de 2015

\section{RESUMEN:}

Los docentes deben hacer uso de las nuevas tecnología para poder realizar un trabajo eficaz en la actual sociedad, donde los procesos educativos están basados en una cada vez mayor cantidad de información y conocimientos.

La formación inicial de estos futuros docentes debe estar impregnada de experiencias enriquecidas con las TIC.

El estudio que se ofrece a continuación pretende conocer y analizar la percepción del alumnado de la Facultad de Ciencias de la Educación de la Universidad de Málaga en relación con la adquisición de las competencias profesionales, referentes a las nuevas tecnologías, necesarias para el desempeño laboral de su profesión.

La investigación es descriptiva y la recogida de información se realiza a través de un cuestionario online realizado y validado previamente para los alumnos de los distintos estudios de grado, licenciaturas y Másteres Oficiales de Postgrado de Ciencias de la Educación.

Los datos y resultados obtenidos son relevantes para determinar nuevas estrategias que permitan la adecuación entre las demandas laborales y la formación académico-profesional adquirida durante los estudios universitarios.

PALABRAS CLAVE: COMPETENCIAS BASADAS EN EDUCACIÓN, EVALUACIÓN DE ESTUDIANTES DE MAESTRO,
COMPETENCIAS, FOMENTO DE LA CAPACIDAD, EDUCACIÓN TECNOLÓGICA.

\section{ABSTRACT:}

Teachers should make use of the new technology to carry out effective work in today's society, where educational processes are based on an everincreasing amount of information and knowledge.

The initial training of these future teachers must be impregnated with experiences enriched with ICT.

The study offered below aims to understand and analyze the perceptions of students of the Faculty of Education Sciences of the University of Malaga, in relation to the acquisition of professional skills, concerning new technologies, necessary to the job performance of their profession.

The research is descriptive and the collection of information is carried out through a questionnaire online performed and validated previously for students of different studies degree, degrees and masters official of postgraduate of Education Sciences.

Data and results obtained are relevant to determine new strategies that will allow the adequacy between the labour demands and the academic and professional training acquired during the university studies.

KEYWORDS: COMPETENCY BASED EDUCATION, STUDENT TEACHER 
EVALUATION, COMPETENCES, CAPACITY BUILDING, TECHNOLOGY EDUCATION.

\section{FUNDAMENTACIÓN Y OBJETIVOS}

Las TIC han tenido un impacto sobre la educación produciendo una serie de transformaciones dentro de ella (Escudero, 2001). Para Freire (2009):

"La educación, como proceso basado en conocimiento, comunicación e interacciones sociales se ha visto afectada de forma radical por la emergencia de la cultura digital, la cual, a su vez, ha transformado a sus actores, profesores y estudiantes, provocando la necesidad de cambios en las propias instituciones educativas" (p. 2)

Con el comienzo de la entrada en vigor del Espacio Europeo de Educación Superior y la consiguiente reforma de las titulaciones universitarias, nos encontramos ante un momento de reformulación de los modelos educativos orientados hacia el desarrollo de competencias profesionales. Este cambio en el sistema universitario español persigue, como uno de sus objetivos, una mayor adecuación entre los requerimientos profesionales del mercado laboral y la formación impartida en las universidades. (Gil, 2013). Su finalidad principal es elevar el grado de ajuste actual entre las competencias que poseen, la importancia que le conceden a las mismas para su futuro profesional y el ámbito al que quieren dirigir sus esfuerzos para insertarse laboralmente.

Castells (1997) define la Sociedad de la Información como un conjunto de transformaciones económicas y sociales que cambiarían la base material de la sociedad. Uno de los fenómenos más importantes de estas transformaciones es la introducción de las nuevas tecnologías de la información y la comunicación en todos los ámbitos de nuestra vida.

Estamos sumergidos en un ambiente de evolución y de cambio, donde el dinamismo del entorno a nivel mundial nos plantea retos, exigencias y oportunidades variadas; nos demanda nuevas y renovadas habilidades, destrezas $\mathrm{y}$ actitudes para enfrentar el ritmo de la modernidad, en donde la innovación y la adaptación al cambio ya no son una opción o un privilegio de algunos, sin constituir una cuestión de interés común y de supervivencia en el escenario de competencia internacional.

La gestión del desempeño por competencias se enfoca esencialmente en el desarrollo, en lo que las personas "serán capaces de hacer" en el futuro.
(Hernández \& otros, 2004). También en los cuatro pilares de la educación presentados en el informe Delors (1996): aprender a conocer, aprender a hacer, aprender a convivir y aprender a ser.

De los profesionales de la educación se pide que sea un intelectual, un guía cultural, un animador, un organizador, un mediador intercultural, etc., y además que desarrolle unas competencias profesionales. Entendemos por competencia la capacidad general o potencialidad adquirida por una persona, que le permite seguir aprendiendo y utilizar lo aprendido para encarar situaciones y resolver problemas reales (Rul y Cambra, 2007). Ser competente no es solo ser hábil en la ejecución de tareas y actividades concretas, sino más allá de ello, ser capaz de afrontar, a partir de las habilidades adquiridas, nuevas tareas o retos que supongan ir más allá de lo aprendido (Monereo y Pozo, 2007).

Según Conner (2013), el aprendizaje en la sociedad del conocimiento se produce en una diversidad de contextos, que van desde los formales a los informales, y desde los intencionales a los inesperados

La gráfica de la compañía Gartner indica fluctuaciones en la aceptación de cualquier Nueva Tecnología: lanzamiento de la tecnología, pico de expectativas sobredimensionadas de la misma, abismo de desilusión, rampa de consolidación y meseta de productividad (ver Figura 1).

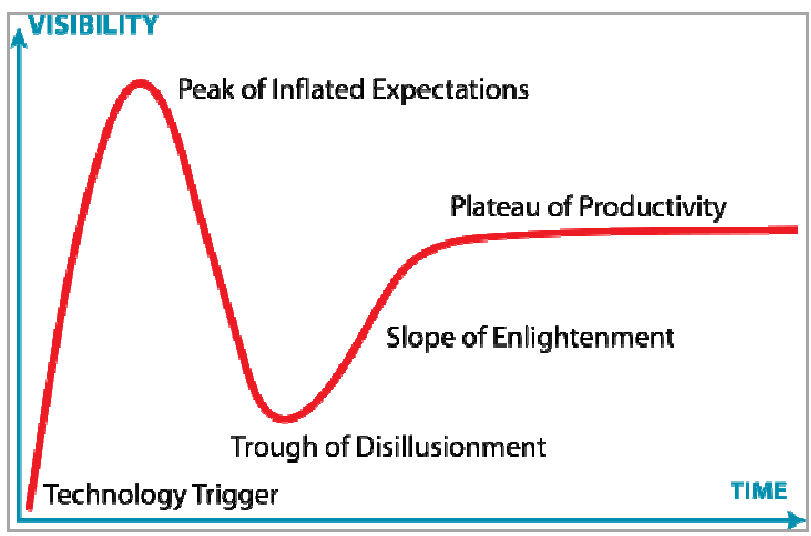

Figura 1. Ciclo de sobreexpectación de las tecnologías según Gartner.

Fuente:

http://en.wikipedia.org/wiki/File:Gartner_Hype_Cycle.svg)

Peláez y Posada (2013), expresan que la utilización de las nuevas tecnologías "se centra en obtener significado de la experiencia de aprendizaje con los demás". "El alumnado es cada vez más autodidacta, aprenden y desaprenden en la potenciación de la interacción y la colaboración con otros". 
Martí, (2012), indica que las contribuciones y discusiones que se realizan en foros virtuales de las asignaturas, planteados tanto por el profesorado como por el alumnado, favorecen la construcción del conocimiento.

La inserción de las tecnologías de la información y de la comunicación (TIC) a lo largo de la última década ha supuesto un cambio en la educación, produciendo un gran impacto en el sistema educativo (creación de infraestructuras, formación del profesorado, etc.) (Almerich y otros, 2011).

\section{PLANTEAMIENTO DEL PROBLEMA O TEMA OBJETO DE ESTUDIO}

El profesorado reconoce que una barrera para la integración de las TIC en su práctica educativa la constituye su propia falta de conocimientos o habilidades (Banlankast and Blamire, 2007; Hew and Brush, 2007; Mueller, Wood, Willoughby, Ross and Specht, 2008; Sigalés et al., 2008). Una elevada proporción de profesorado se autoevalúa como no suficientemente capacitados para utilizar las TIC (Cabero, 2004). Esta constatación explica que el profesorado siga demandando formación en la integración de estos nuevos recursos educativos en su labor docente (Rudd et al., 2009).

También es interesante conocer la disposición y el grado de utilización de las TIC por el alumnado universitario. Una parte del alumnado puede que en la práctica realicen solo las tareas obligatorias, sin implicarse en todas las posibles actividades asociadas a las TIC (foros, acciones de trabajo colaborativo,...); y otra parte del alumnado puede mostrarse activo en la utilización de las TIC. Según Hill (2012), se trata de alumnado que presenta un alto índice de participación en las actividades propuestas por el profesorado, intentando aprovechar al máximo las posibilidades de la utilización de las TIC como instrumento de aprendizaje para la adquisición de competencias en el ámbito académico. Por ello, consideramos importante conocer la percepción de los estudiantes sobre la importancia del uso de las TIC en su proceso de formación, si favorece su participación activa y el intercambio de información, es decir, la interacción alumnado-alumnado y alumnadoprofesorado. Ambos aspectos son claves para el aprendizaje de competencias.

Asimismo, consideramos fundamental, avanzar en la percepción del alumnado universitario en la necesidad de alcanzar un elevado nivel de competencias tecnológicas e instrumentales para su inserción efectiva en el ámbito laboral. El uso efectivo de las TIC puede favorecer la competencia digital, sobre todo en relación con el fomento de la autonomía en el proceso de aprendizaje y en la autorregulación del aprendizaje (Vázquez \& Sevillano, 2011; Cabero, Marín \& Llorente, 2012; Cabero, 2013).

En la actualidad existe un déficit en la realización de estudios para analizar la percepción del alumnado universitario sobre las posibilidades de mejora competencial que ofrecen las TIC, de sus limitaciones y problemáticas en el uso de las nuevas tecnologías y sus aplicaciones, cada vez más relevantes en el mundo laboral.

Ante esta situación, el presente estudio tiene como objetivo principal conocer y analizar la percepción del alumnado de la Facultad de Ciencias de la Educación de la Universidad de Málaga en relación con la adquisición de las competencias relacionadas con la utilización de las TIC, es decir, si consideran que poseen un perfil competencial adecuado en relación con las TIC para desarrollar una óptima formación académica y también para aumentar las posibilidades de su posterior inserción laboral.

\section{OBJETIVO E HIPÓTESIS}

El objetivo formulado para este trabajo es el siguiente: Conocer y analizar la percepción del alumnado de la Facultad de Ciencias de la Educación de la Universidad de Málaga en relación con la adquisición de las competencias profesionales, referentes a las nuevas tecnologías, necesarias para el desempeño laboral de su profesión docente.

La hipótesis de trabajo que se ha formulado es: Los estudiantes de las titulaciones de la Facultad de Ciencias de la Educación de la Universidad de Málaga poseen las capacidades necesarias para el desempeño de su futura profesión docente.

\section{DISEÑO Y METODOLOGÍA}

\subsection{Instrumento}

El equipo investigador del presente estudio diseñó un cuestionario inicial en base a una batería de preguntas relacionadas con la percepción del alumnado universitario sobre el grado de adquisición de capacidades básicas para su desarrollo profesional.

El cuestionario inicial elaborado constaba de 33 preguntas organizadas en dos bloques diferentes. Un primer bloque de cuestiones referidas a los datos 
demográficos del alumnado participante $\mathrm{y}$ un segundo bloque de contenidos relacionado con las competencias universitarias y la utilización de las TIC.

Se utilizó un vocabulario específico relacionado con las destrezas básicas de las profesiones relacionadas con la educación, todos los términos totalmente conocidos por el alumnado, no incluyendo ninguna abreviatura aunque nos resultara especialmente conocido el término.

Utilizamos una escala de respuestas tipo Likert sobre el grado de acuerdo sobre las cuestiones planteadas. Cada ítem se refiere a una capacidad concreta y debe ser contestado en base a una escala del 1 al 5 donde:

1. Capacidad no adquirida.

2. Capacidad poco adquirida.

3. Capacidad adquirida en parte.

4. Capacidad bastante adquirida.

5. Capacidad completamente adquirida.

Para el estudio preliminar y validación del cuestionario como instrumento adecuado para los objetivos de la investigación. En primer lugar, solicitamos la colaboración de diez profesores del Departamento de Métodos de Investigación e Innovación Educativa de la Facultad de Ciencias de la Educación de la Universidad de Málaga para realizar una encuesta piloto y comprobar el adecuado grado de comprensión de las cuestiones planteadas,

Una vez finalizada la revisión del cuestionario, este fue validado por un comité de jueces formado por cinco profesores expertos en la materia de diferentes

Departamentos de la Facultad de Ciencias de la Educación. El citado comité evaluó y valoró positivamente la relación existente entre los objetivos de nuestro estudio y los ítems reflejados en el cuestionario.

\subsection{Muestra}

Hemos realizado un tipo de muestreo no probabilístico accidental (Buendía, Colás y Hernández, 1998). Hemos seleccionado 73 alumnos de la Facultad de Ciencias de la Educación como muestra de nuestro estudio, se trata de alumnado de diferentes asignaturas de la facultad de Ciencias de la Educación de la Universidad de Málaga.

Con respecto a las edades de nuestra muestra un $68 \%$ de la misma está comprendida "entre los 20 y los 25 años de edad", siendo el resultado de "más de 30 años" del $12 \%$. De estos participantes el $81 \%$ son mujeres mientras que el $19 \%$ son hombres.

\subsection{Análisis estadístico}

Una vez recogidos los cuestionarios, los datos fueron analizados mediante el programa de análisis estadístico IBM SPSS Statistics versión 20.

\subsection{Fiabilidad del instrumento}

Con el fin de hallar la fiabilidad del instrumento se utilizó el Coeficiente Alfa de Cronbach. Este instrumento es uno de los más utilizados para establecer la fiabilidad de cuestionarios o escalas; está basado en la consistencia interna del mismo (Vilanova et al. 2007). El coeficiente que brinda depende, tanto del número de ítems del instrumento, como de la correlación entre los mismos o sus covarianzas; sus valores deben ubicarse entre $0.0 \mathrm{y}$ 1.0 , y se consideran valores aceptables a partir del 0.7. De esta manera, se calculó el Alfa de Cronbach obteniéndose un valor medio de 0,855 en las 25 variables del bloque de contenido de la encuesta, lo cual significa que el cuestionario tiene una alta fiabilidad.

\section{RESULTADOS}

Debido a la integración de las TIC en educación, se originan unas consecuencias por parte de la institución universitaria:

- En el rol de profesores, que cambian de ser transmisores del conocimiento al alumnado a ser mediadores en la construcción del propio conocimiento por parte de estos (Salinas, 1999). Según Feixa, Márquez y Tómas (1999) la Universidad ante los procesos de cambio científico, tecnológico y social debe prestar atención a la innovación para el cambio, aplicar las TIC y desarrollar proyectos de formación permanente para el personal. Para ello además de proporcionar los recursos tecnológicos necesarios y el apoyo a la docencia en cuanto a su utilización, deberá impulsar planes de formación necesarios en cuanto a formación en uso de los nuevos recursos tecnológicos, modelación de actitudes positivas hacia las TIC, las metodología didácticas centradas en el aprendizaje y la aplicación de técnicas de investigación-acción en el aula y formación en metodologías para el aprovechamiento de las TIC.

- En el rol del alumno quien en contacto con las TIC deberá desarrollar una serie de acciones educativas relacionadas con el uso, la selección utilización y organización de la información. Será un participante activo del proceso de 
aprendizaje, él mismo deberá producir y compartir el conocimiento, a veces participando como experto, dentro de un marco colaborativo de aprendizaje (Silva, 2007). El apoyo y orientación que reciba por parte del profesor será clave en el aprovechamiento de las TIC (Salinas, 2004).

- En los aspectos metodológicos, según Feixa, Márquez y Tómas (1999) las TIC provocan los siguientes cambios en la docencia:

- Nuevos contenidos y competencias en el currículo. Las TIC constituyen un contenido transversal en todas las áreas de conocimiento que hace necesaria la renovación de los programas de las asignaturas.

- Nuevos instrumentos para la docencia y su gestión tanto offline como online.

- Nuevos métodos pedagógicos. Hoy en día los procesos de enseñanza y aprendizaje se basan en perspectivas socio-constructivistas que otorgan gran importancia a la actividad de los estudiantes y su interacción con el contexto.

Nos vamos a centrar en nuestro estudio en las consecuencias que afectan al alumnado. Por ello hemos revisado las programaciones de las titulaciones implicadas en nuestro estudio para conocer si se ven reflejadas en las competencias a desarrollar en el alumnado. Hemos encontrado poca presencia de estas en las competencias específicas de cada titulación. Algunas de estas han sido:

- Competencias específicas del grado de primaria: Valorar la trascendencia de las ciencias como un hecho cultural y la necesidad de la alfabetización científica y tecnológica de la ciudadanía, reconociendo y valorando la mutua influencia.

- Competencias Grado de Pedagogía: Promover, planificar y gestionar la implantación de procesos de innovación educativa y de modelos de gestión de la calidad.

- Competencias en el Máster de Secundaria: Conocimiento de informática en el ámbito de estudio

Basándonos en los datos encontrados pasamos a analizar los resultados conseguidos.

En los cuestionarios suministrados a nuestra muestra de estudio encontramos las capacidades profesionales que los alumnos adquieren en los estudios universitarios que cursan.
El análisis de los datos se ha realizado según las cuatro capacidades que opinamos están relacionadas con las nuevas tecnologías.

1. Capacidad para manejar las nuevas tecnologías.

2. Capacidad para aprender nuevos métodos de trabajo.

3. Capacidad para el autoaprendizaje.

4. Capacidad de utilización de diferentes recursos didácticos (pizarras digitales, vídeos, etc.).

En cuanto a la información demográfica logramos extraer que la mayoría del alumnado participante son de género femenino (81\%) frente al masculino (19\%), y aunque es mayor el porcentaje de participantes del Grado de Maestro, donde se incluye tanto Primaria como Infantil (un 34\%), estos no distan mucho del porcentaje del Grado de Pedagogía (un 30\%) siendo el resto de participantes de las antiguas titulaciones de Psicopedagogía (7\%), Pedagogía(10\%) y Máster (7\%).

Las siguientes respuestas están basadas en una escala de respuesta tipo Likert de 5 puntos donde 1 es capacidad no adquirida, 2 es capacidad poco adquirida, 3 capacidad adquirida en parte, 4 capacidad bastante adquirida y 5 capacidad completamente adquirida.

Con respecto a la capacidad que poseen los alumnos para el manejo de las nuevas tecnologías, comenzamos destacando que no tienen adquirida esa capacidad solo el $1 \%$ o la tienen poco adquirida el $4 \%$. Sin embargo, el $40 \%$ la tiene completamente adquirida y el $38 \%$ bastante adquirida.

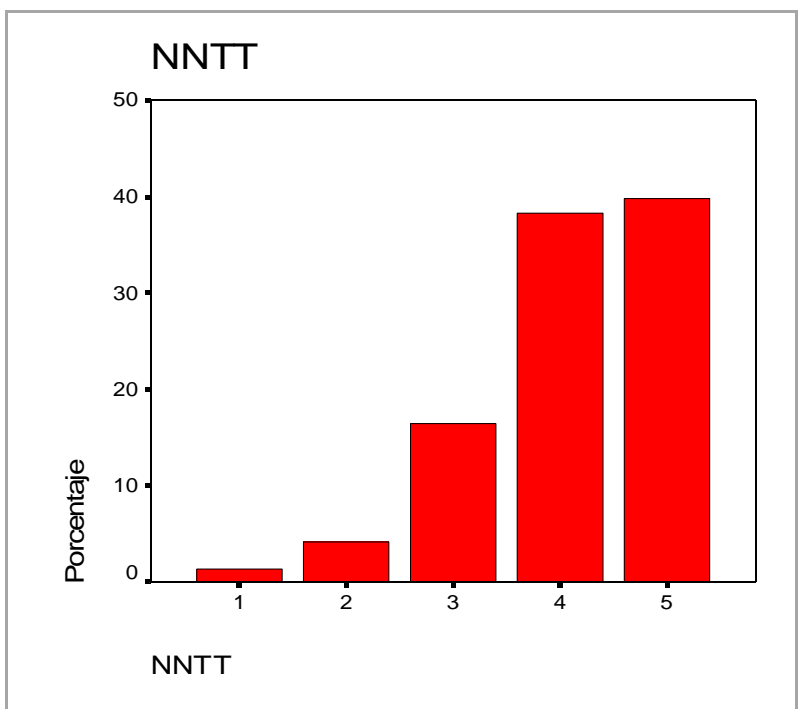

Figura 2. Capacidad para manejar las nuevas tecnologías. Fuente: elaboración propia

En cuanto al manejo de las nuevas tecnologías la mayoría de los encuestados están capacitados ya que 
casi la mitad tienen completamente adquirida esta competencia.

Centrándonos en la capacidad para el aprendizaje de nuevos métodos de trabajo destaca que no existe ningún alumno que no tenga adquirida esta. Únicamente un $3 \%$ indica que la tiene poco adquirida frente a un $58 \%$ que la tiene bastante adquirida o un $26 \%$ que la poseen completamente.

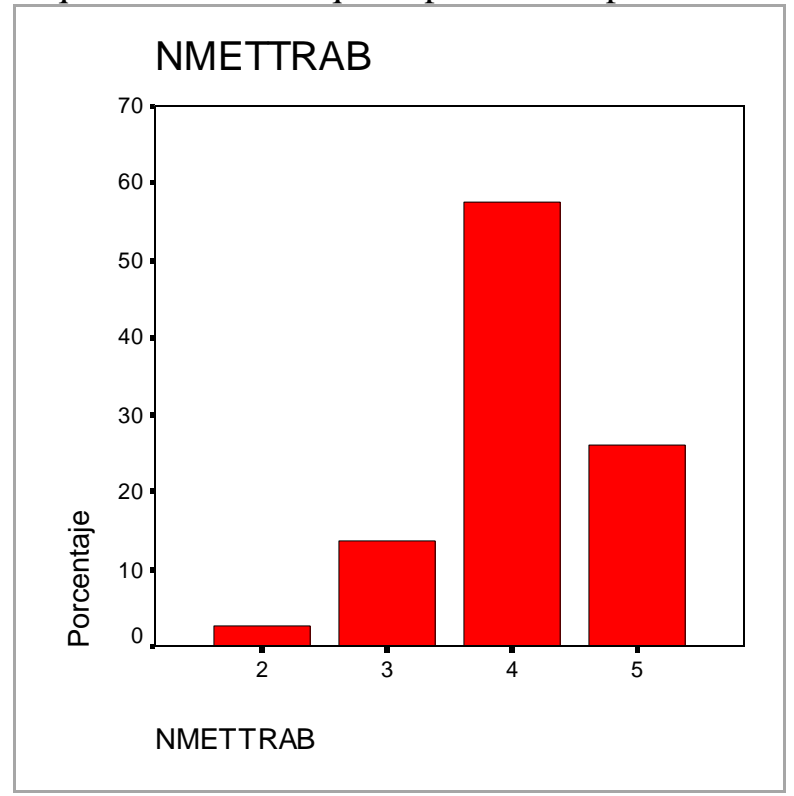

Figura 3. Capacidad para aprender nuevos métodos de trabajo. Fuente: elaboración propia

Podemos observar que, a diferencia de la primera capacidad, en esta el mayor porcentaje está entre el alumnado que tiene bastante conocimientos sobre nuevos métodos de trabajo, pero que considera que aún le quedan por aprender.

De igual modo, la capacidad para el autoprendizaje la tienen completamente adquirida el $26 \%$ de los participantes y un $47 \%$ la tienen bastante adquirida mientras el $3 \%$ solo la tienen un poco adquirida y $1 \%$ no la tiene adquirida.

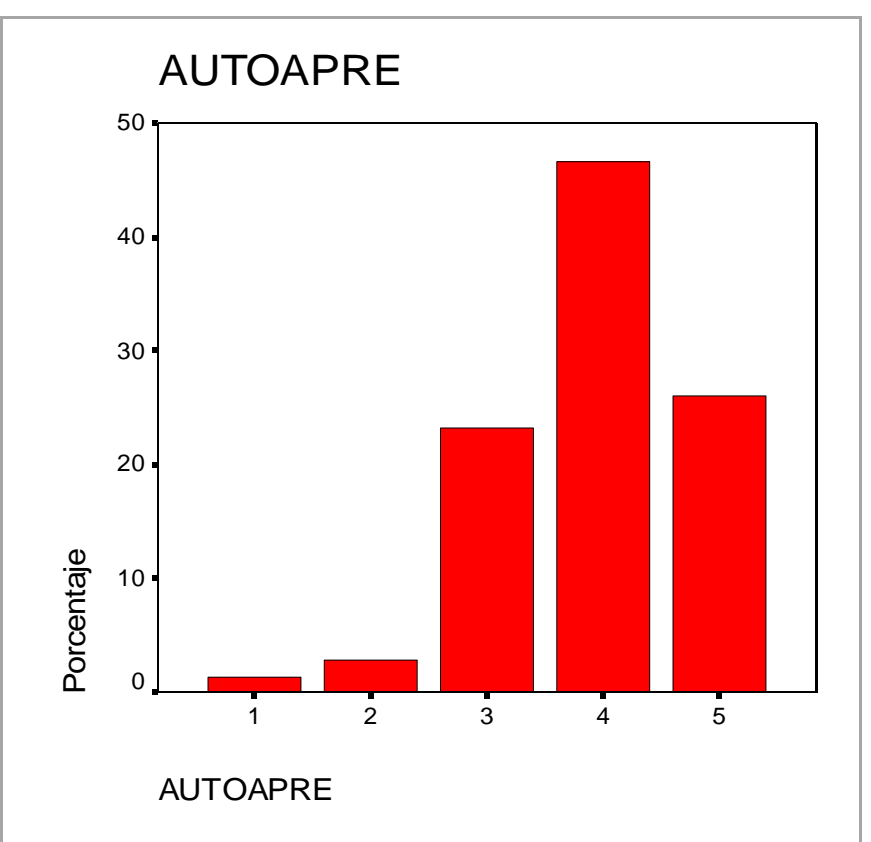

Figura 4. Capacidad para el autoaprendizaje Fuente: elaboración propia

En este caso también se hace latente la buena capacidad para el autoaprendizaje adquirido por nuestros alumnos pero la falta a su vez de un número de habilidades al respecto.

En relación a la capacidad de uso de los diferentes recursos didácticos (pizarras digitales, vídeos, etc) respondieron que no la tenían adquirida el $4 \%$ y poco adquirida el $5 \%$. Por otro lado, el $42 \%$ del alumnado opina que la tiene bastante adquirida y el $30 \%$ la tiene completamente adquirida.

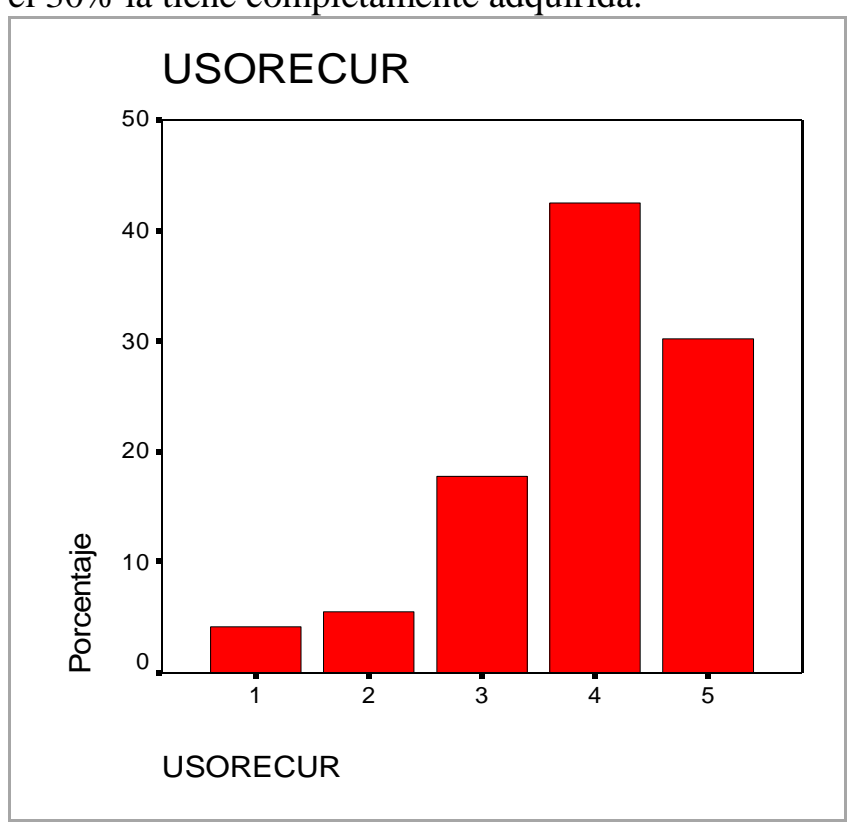

Figura 5. Capacidad de utilización de diferentes recursos didácticos

Fuente: elaboración propia 
La valoración que la mayoría de los estudiantes otorgan a la capacidad de uso de los diferentes recursos didácticos tecnológicos es considerable ya que opinan que tienen bastante adquirida esta competencia a pesar de que también les quede por aprender.

\section{DISCUSIÓN}

Teniendo en cuenta los resultados obtenidos en los cuestionarios podemos concluir lo siguiente:

1. Un gran porcentaje del alumnado está capacitado para manejar las nuevas tecnologías mientras que, en cambio, hay un bajo porcentaje que la tiene poco o no la tiene adquirida. Esto nos indica que en los estudios universitarios analizados se está dando una formación adecuada a este respecto.

2. Respecto a la capacidad para aprender nuevos métodos de trabajo, entendidos estos métodos para aplicar las nuevas tecnologías a la hora de enseñar a sus futuros alumnos, los resultados muestran que existe un alto porcentaje que tiene bastante $\mathrm{o}$ completamente adquirida esta capacidad.

3. En cuanto a la capacidad de autoaprendizaje por parte del alumnado estos indican que en su mayoría tiene completamente o bastante adquirida la misma para ser competente en su futura profesión docente.

4. Finalmente, las capacidades de utilización de diferentes recursos didácticos, entendiendo estos los relacionados con las nuevas tecnologías como la pizarra digita, vídeos, televisores, ordenadores, móviles, libros digitales, etc., están bastante o completamente adquiridas en un $73 \%$ frente a un 9\% que la tiene poco o no la tiene adquirida.

El análisis de los resultados que ofrece la presente investigación nos ha permitido dar respuesta a los objetivos planteados y dar por válida nuestra hipótesis de trabajo.

La investigación revela que, a pesar de que, en los programas de las diferentes titulaciones estudiadas, no se reflejan lo suficiente las competencias específicas relacionadas con el aprendizaje y manejo de las nuevas tecnologías, finalmente podemos concluir, que en las especialidades estudiadas, se está dotando al alumnado de las competencias básicas que estos van a necesitar en un futuro profesional en lo que respecta a las nuevas tecnologías.

No obstante, estos resultados obtenidos en nuestro estudio no coinciden con otros estudios encontrados, en donde los resultados obtenidos en las necesidades formativas en TIC concuerdan con las competencias y uso de estos recursos por parte del profesorado. Así, el profesorado presenta un nivel bajo en las competencias en TIC, dominando más las competencias tecnológicas que las pedagógicas. El uso de estas tecnologías en general es bajo, si bien el plano personal-profesional presenta un nivel más alto respecto a la utilización en el aula. El uso de las TIC con el alumnado en el aula es muy limitado y más bien ocasional (Aguaded y Tirado, 2010, Valcke et al., 2007) (Almerich, 2011).

\section{REFERENCIAS}

Almerich, G., Suárez-Rodríguez, J. M., Belloch, C., \& Bo, R. M. (2011). Las necesidades formativas del profesorado en TIC: perfiles formativos y elementos de complejidad. Relieve, 17(2). Recuperado de http://www.uv.es/RELIEVE/v17n2/RELIEVEv17n2_1. htm

Balanskat, A., y Blamire, R. (2007). ICT in Schools: Trends, Innovations and Issues in 2006-2007. European Schoolnet. Consultado en

http://insight.eun.org/shared/data/pdf/ict_in_schools_20067_final.pdf el 20 de mayo de 2009.

Balanskat, A., Blamire, R., \& Kefala, S. (2006). The ICT impact report. A review of studies of ICT impact on schools in Europe. European Schoolnet. Consultado en http://insight.eun.org/shared/data/pdf/impact_study.pdf el 5 de diciembre de 2008.

Cabero, J. (2004). Formación del profesorado en TIC. El gran caballo de batalla. Comunicación y Pedagogía: Nuevas tecnologías y recursos didácticos, 195, 27-31.

Cabero, J., y Marín, V. (2012). La capacitación en TIC del profesorado universitario en un Entorno Personal de Aprendizaje. El proyecto DIPRO 2.0. New approaches in Educational Research, 1, 2-7. Disponible en http://naerjournal.ua.es/article/view/9.

Cabero, J., \& Marín, V. (2013). Percepciones de los estudiantes universitarios latinoamericanos sobre las redes sociales y el trabajo en grupo. Revista de Universidad y Sociedad del Conocimiento (RUSC), 10(2), 219-235. Disponible en http://rusc.uoc.edu/ojs/index.php/rusc/article/view/v10n 2-cabero-marin/v10n2-cabero-marin-es.

Castells, M. (1997). La era de la informática. Economía, sociedad y cultura. La Sociedad Red. Madrid: Alianza.

Conner, M.L. (2013). Informal Learning. Disponible en http://marciaconner.com/resources/informal-learning/.

Delors, J. (1996). La educación encierra un tesoro. Madrid: Santillana.

Escudero, J. M. (2001). La educación y la sociedad de la información: cuestiones de contexto y bases para un diálogo necesario. En F. Blázquez (Coord.), Sociedad de la Información y la Comunicación. Mérida: Consejería de Educación, Ciencia y Tecnología.

Feixas, M., Márques, P., y Tomás, M. (1999). La Universidad ante los retos que plantea la sociedad de la información. El papel de las TIC. En J. Salinas (Coord.), Actas de las Jornadas EDUTEC-99. Rol del profesorado universitario ante los cambios de la era digital. Perfeccionamiento Integral del Profesor Universitario, 
Primer Encuentro Iberoamericano. Caracas: Universidad Central de Venezuela.

Freire, J. (2009). Monográfico cultural digital y prácticas creativas en educación. RUSC. Revista De Universidad y Sociedad Del Conocimiento, 6(1).

Salinas, J. (2004). Innovación docente y uso de las TIC en la enseñanza universitaria. Revista de Universidad y Sociedad del Conocimiento, 1(1), 1-16.

Hernández, M., Cuétara, L., Frías, R., \& Pigueira, R. (2004). Evaluación de competencias básicas de profesionales universitarios en la actividad hotelera.

Recuperado de http://revistas.mes.edu.cu/greenstone/collect/repo/impor t/repo/20090820/16819713006.pdf

Hew, K. F., \& Brush, T. (2007). Integrating technology into K12 teaching and learning: current knowledge gaps and recommendations for future research. Educational Technology Research Development, 55(3), 227-243.

Hill, P. (2012). Four Barriers that MOOCs must overcome to build a sustainable model. e-Literate. Recuperado de http://mfeldstein.com/four-barriers-that-moocs-mustovercome-to-become-sustainable-model/.

Martí, J. (2012). Tipos de MOOCs. Xarxatic. Disponible en http://www.xarxatic.com/tipos-de-moocs/.

Monereo, C., y Pozo, J. I. (2007). Competencias para (con)vivir con el siglo XXI. Cuadernos de Pedagogía, 370, 12-18.

Mueller, J., Wood, E., Willoughby, T., Ross, C., \& Specht, J. (2008). Identifying discriminating variables between teachers who fully integrate computers and teachers with limited integration. Computers \& Education, 51(4), 1523-1537.

Peláez, A.F., \& Posada, M. (2013). Autonomía en Estudiantes de Posgrado que participan en un MOOC. Caso Universidad Pontificia Bolivariana. En SCOPEO Informe n². MOOC: Estado de la situación actual, posibilidades, retos y futuro (pp. 174-193). Salamanca: Universidad de Salamanca-Centro Internacional de Tecnologías Avanzadas.

Programas de titulaciones de la Facultad de Ciencias de la Educación de la Universidad de Málaga. (2015) Disponible en http://www.uma.es/centroslistado/info/4421/facultad-de-ciencias-de-la-educacion/

Rul, J., y Cambra, T. (2007). Educación y competencias básicas. Cuadernos de Pedagogía, 370, 71-80.

Sigalés, C., Mominó, J. M., Meneses, J. y Badía, A. (2008). La integración de internet en la educación escolar española: situación actual y perspectivas de futuro. Barcelona: UOC. Recuperado de http://www.fundacion.telefonica.com/debateyconocimie nto/publicaciones/informe_escuelas/esp/pdf/informe_es cuelas.pdf 